\title{
Mecanismos de desconexión moral y su relación con la empatía y la prosocialidad en adolescentes que han tenido experiencias delictivas
}

\author{
Anyerson Stiths Gómez Tabares ${ }^{1}$, Mariela Narváez Marín ${ }^{2}$ \\ Universidad Católica Luis Amigó-Colombia ${ }^{1}$,Universidad de Manizales-Colombia ${ }^{2}$
}

\begin{abstract}
Se buscó identificar la relación entre los mecanismos de desconexión moral, la empatía y la prosocialidad en adolescentes que han tenido experiencias delictivas. Se contó con una muestra de 60 adolescentes que contaron con entre 16 a 18 años, a quienes se les aplicó el cuestionario de desconexión moral (MMD), la escala de tendencias prosociales (PTM-R) y el índice de reactividad emocional (IRI). Se encontró que el mecanismo de desconexión moral con mayor puntuación fue justificación moral. Asimismo, la desconexión moral correlacionó negativamente con las conductas prosociales y la empatía. Las mujeres presentan promedios más altos en las dimensiones de la empatía y la prosocialidad, mientras que los hombres presentan promedios más altos en los mecanismos de desconexión moral. Se concluye que es importante generar estrategias que estimulen la empatía, la solidaridad, la educación emocional y criterios morales prosociales en adolescentes infractores de la ley. Palabras clave: adolescente, conducta prosocial, desconexión moral, empatía, delito, agresividad.
\end{abstract}

Mechanisms of moral disconnection and its relationship with empathy and prosociality in adolescents who have had criminal experiences

We sought to identify the relationship between the mechanisms of moral disconnection, empathy and prosociality in adolescents who have had criminal experiences. A sample of 60 adolescents between 16 and 18 years old answered the moral disconnection questionnaire (MMD), the prosocial tendency scale (PTM-R) and the emotional reactivity index (IRI). The moral disconnection mechanism with the highest score was moral justification. Moral disconnection negatively correlated with prosocial behaviors and empathy. Women presented higher means in the dimensions of empathy and prosociality, while men showed higher means in the mechanisms of moral disconnection. The study concludes that it is important to generate strategies that stimulate empathy, solidarity, emotional education and prosocial moral criteria in adolescents who break the law.

Keywords: adolescent, prosocial behavior, moral disconnection, empathy, crime, aggressiveness.

1 Magíster en Educación, UNINI. Docente e investigador adscrito al Programa de Psicología de la Universidad Católica Luis Amigó, Regional Manizales, Colombia. Contacto: anyerspn. gomezta@amigo.edu.co

2 Licenciada en Ciencias Sociales, UTP. Especialista en Farmacodependencia, FUNLAM. Doctoranda en Salud Pública, Universidad Miguel Hernández, España. Docenteinvestigadora de Universidad de Manizales, Colombia. Contacto: maye888@gmail.com 


\section{Mecanismos de desconexáo moral e sua relaçáo com empatia e prosocialidade em ado- lescentes que tiveram experiências criminosas}

Procurou-se identificar a relação entre os mecanismos de desconexão moral, empatia e pró-socialidade em adolescentes que tiveram experiências criminosas. Contou-se com uma amostra de 60 adolescentes com idades entre os 16 e os 18 anos, aos quais foram aplicados o questionário de desconexão moral (DMM), a escala de tendência pró-social (PTM-R) e o índice de reatividade emocional (IRI). Descobriu-se que o mecanismo de desconexão moral com a maior pontuação era justificativa moral. Da mesma forma, a desconexão moral correlacionou-se negativamente com comportamentos pró-sociais e empatia. As mulheres apresentam médias mais elevadas nas dimensōes de empatia e pró-socialidade, enquanto os homens apresentam maiores médias nos mecanismos de desconexão moral. Conclui-se que é importante gerar estratégias que estimulem a empatia, a solidariedade, a educação emocional e os critérios morais pró-sociais nos adolescentes infratores da lei.

Palavras-chave: adolescente, comportamento pró-social, desconexão moral, empatia, crime, agressividade

\section{Mécanismes de déconnexion morale et sa relation avec l'empathie et la prosocialité chez les adolescents ayant eu des expériences criminelles}

Nous avons cherché à identifier la relation entre les mécanismes de déconnexion morale, d'empathie et de prosocialité chez les adolescents ayant vécu des expériences criminelles. Un échantillon de 60 adolescents âgés de 16 à 18 ans a appliqué le questionnaire de déconnexion morale (MMD), l'échelle de tendance prosociale (PTM-R) et l'indice de réactivité émotionnelle (IRI). Il a été constaté que le mécanisme de déconnexion morale avec le score le plus élevé était une justification morale. De même, la déconnexion morale a une corrélation négative avec les comportements prosociaux et l'empathie. Les femmes présentaient des moyens plus élevés dans les dimensions de l'empathie et de la prosocialité, tandis que les hommes montaient des moyens plus élevés dans les mécanismes de déconnexion morale. Létude conclut qu'il est important de générer des stratégies qui stimulent l'empathie, la solidarité, l'éducation émotionnelle et les critères moraux prosociaux chez les adolescents qui enfreignent la loi.

Mots-clés: adolescent, comportement prosocial, déconnexion morale, empathie, crime, agressivité 
La desconexión moral ha sido un tema de creciente interés investigativo en las ciencias sociales, especialmente en lo que concierne a la comprensión de la conducta agresiva y antisocial en la infancia y la adolescencia (Gini, Pozzoli \& Bussey, 2015; Kokkinos \& Kipritsi, 2017; Gini, Pozzoli \& Hymel, 2014), justamente porque se ha considerado que la conducta violenta y delincuencial requiere del uso de estrategias cognitivas para no dar cuenta de las normas y códigos ético-morales impuestos por la sociedad, y, de esta manera, evitar la autocensura (Giulio, Petruccelli \& Pace, 2018). En este sentido, la desconexión moral se considera un constructo psicológico de gran importancia para comprender los procesos cognitivo-morales asociados a la conducta delictiva, más allá de la simple descripción de las características sociales y psicológicas del infractor (Giulio et al., 2018; Petruccelli et al., 2017). Para Gutzwiller-Helfenfinger (2015), mediante el uso de uno o varios mecanismos de desconexión moral, la conducta perjudicial por parte del infractor se reconstruye cognitivamente para que parezca menos perjudicial para sí mismo y lo demás, y así justificar la acción.

Para Bandura (1990, 1991, 1999, 2002) el comportamiento moral está regido por mecanismos cognitivos de autorregulación, los cuales le permiten al ser humano actuar de manera correcta o incorrecta, moral o inmoralmente, de acuerdo con los estándares éticos a nivel social. La desconexión moral, en este sentido, es un proceso de reconstrucción cognitiva o reformulación de la conducta violenta o nociva como algo moralmente aceptable, lo que le permite a la persona evitar la autocensura y la culpa cuando actúa en contravía a los criterios ético-morales dentro de un sistema social (Bandura, 1999; Hymel \& Perren, 2015; Doyle \& Bussey, 2017).

En otras palabras, cuando la persona se desconecta moralmente no se activan los procesos regulatorios a nivel cognitivo y emocional relacionados con la autoevaluación negativa de los actos o comportamientos 
violentos, agresivos o delincuenciales, evitando así la culpa, la vergüenza y la autocensura (Bandura, 1999; Bandura et al., 1996; Gini et al., 2014; Hymel \& Perren, 2015). De acuerdo con Kokkinos \& Kipritsi (2017), y Gini, Pozzoli y Hauser (2011) la desconexión moral funciona como una estrategia sociocognitiva y de autorregulación mediante la cual la persona justifica las conductas moralmente incorrectas, y así evitan la disonancia cognitiva entre su sentido moral y los estándares éticos a nivel social. Para Bandura (1990):

Las autosanciones pueden ser desactivadas reconstruyendo la conducta perjudicial a través de la justificación moral, el etiquetado eufemístico y el contraste ventajoso con otras inhumanidades; ocultando la agencia personal en actividades perjudiciales a través de la difusión y el desplazamiento de la responsabilidad; al ignorar o tergiversar las consecuencias dañinas de la conducta inhumana; y culpando y deshumanizando a las víctimas (p. 27).

La desconexión moral, según Bandura (1990, 1991, 2002), se ha caracterizado en ocho mecanismos: justificación moral (es decir, la persona busca validar para sí misma la moralidad de una acción inmoral o incorrecta); comparación ventajosa (consiste en agrandar otras conductas inhumanas para que la propia conducta moralmente incorrecta parezca menos perjudicial o incluso benevolente); lenguaje eufemistico (las acciones pueden tomar otra apariencia dependiendo de cómo se llamen. La persona hace uso de un lenguaje que modere y disminuya la importancia del comportamiento censurado); desplazamiento de la responsabilidad (ocultar o minimizar la conducta realizada a través de la atribución de la responsabilidad a otras personas o una autoridad legítima, en lugar de asumir la responsabilidad de las acciones); difusión de la responsabilidad (cualquier daño hecho en grupo siempre puede ser atribuido en gran parte al comportamiento de otros. Las personas actúan más cruelmente cuando existe una responsabilidad grupal que cuando se hacen personalmente responsables de su comportamiento); distorsión de las consecuencias (los daños ocasionados por una conducta se ignoran, malinterpretan o minimizan, evitando que se active la autocensura moral); deshumanización (considerar a la persona a la cual se 
le hace daño como carente de humanidad, salvaje, cruel o desalmada; en últimas, despojarla de toda humanidad para justificar el daño hacia ella) y, atribución de la culpa (es decir, se consideran a sí mismos como víctimas impulsadas a realizar la conducta perjudicial por una provocación forzosa, atribuyéndole la culpa a los demás).

Diversos estudios (Bandura, Underwood y Fromson, 1975; Caprara, Alessandri, Fida, Tisak, Fontaine \& Paciello, 2014; Gini et al., 2014; Hardy, Bean \& Olsen, 2014; Martínez-Morales, Robles-Haydar, Amar-Amar \& Crespo-Romero, 2016; Jolliffe \& Farrington, 2006; Bussey, Quinn \& Dobson, 2015; Gini et al., 2015) se han orientado a investigar la desconexión moral en contextos educativos, encontrando una relación estadísticamente significativa con la conducta agresiva, la intimidación física, verbal y relacional en niños y adolescentes. Cuanto mayor es la conducta de agresión física o verbal, directa o indirecta, mayor es el uso de mecanismos de desconexión moral para justificar acciones consideradas reprobables por la sociedad, y menor es el sentido de empatía y prosocialidad por el bienestar de los demás (Giulio et al., 2018; Kokkinos \& Kipritsi, 2012, 2017; Raskauskas, Gregory, Harvey, Rifshana \& Evans, 2010).

En el metanálisis hecho por Gini et al. (2014) encontraron relación entre desconexión moral y diferentes tipos de conducta agresiva, hallazgos que se explican porque los mecanismos cognitivos que posibilitan la desconexión moral se utilizan ante situaciones o conductas que se consideran moralmente incorrectas por la sociedad, ya sea porque van contra la normatividad institucional o atentan contra el bienestar de otras personas y, de este modo, se evita la autocensura o devaluación sobre la manera en que se actúa.

De otro lado, y de manera más explícita, diversos autores (Bussey et al., 2015; Gini, 2006; Gini et al., 2014, 2015; Pozzoli, Gini \& Vieno, 2012) han relacionado la desconexión moral con el bullying, de tal modo que los mecanismos cognitivos de separación moral inciden en la aparición y mantenimiento de conductas de intimidación escolar, así como en la participación en roles asociados a la legitimación de la violencia, por ejemplo, el rol de espectador u observador. $\mathrm{Al}$ respecto, 
Pozzoli, Gini \& Vieno (2012) evaluaron 38 niños de quinto grado escolar y encontraron relación entre la reestructuración cognitiva y promover el bullying a nivel individual. A nivel grupal encontraron significancia entre minimizar el rol, deshumanizar a la víctima y culpar a otros. El mecanismo de autojustificación fue estadísticamente asociado con el comportamiento individual de bullying.

Desde entonces han sido diversos los campos de estudio en los que se ha demostrado la incidencia de la desconexión moral con diversos comportamientos transgresores, entre ellos, el terrorismo y las actividades bélicas (Bandura, 1988; Villegas de Posada, Flórez \& Espinel, 2018; Bandura, 2004; Osofsky, Bandura \& Zimbardo, 2005), el consumo de drogas y alcohol (Quinn \& Bussey, 2015b, 2015a; Giulio et al., 2018), la propensión de los niños a decir mentiras (Doyle \& Bussey, 2017), el uso de esteroides en el deporte (Lucidi et al., 2008; Boardleya et al., 2018), el rol de la parentalidad en la desconexión moral en la niñez (Campaert, Nocentini \& Menesini, 2018; Hyde, Shaw \& Moilanen, 2010; Houwing \& Bussey, 2017) y, en especial, las actividades delictivas en jóvenes (Giulio et al., 2018; DeLisi et al., 2014; Petruccelli et al., 2017), siendo este último un campo de estudio reciente con múltiples aristas por explorar.

De acuerdo con Shulman, Cauffman, Piquero y Fagan (2011), los adolescentes que presentan puntuaciones más altas en desconexión moral son más propensos a involucrarse en actividades antisociales y delictivas, lo cual es coherente con otras investigaciones (Bandura, Barbaranelli, Caprara \& Pastorelli, 1996; Bandura et al., 2001; Paciello et al., 2008; Pelton, Gound, Forehand \& Brody, 2004; DeLisi et al., 2014; Petruccelli et al., 2017) que muestran una mayor tendencia delictiva y antisocial en adolescentes que evidencian mayores mecanismos cognitivos orientados a la desconexión moral, lo que indica que este constructo psicológico es un predictor importante de la conducta antisocial en curso entre adolescentes y jóvenes delincuentes. Al respecto, el estudio de Giulio et al. (2018) con 30 narcotraficantes y 19 delincuentes varones, muestra que, el primer grupo presentaron niveles más altos en desconexión moral general, así como en los mecanismos 
de deshumanización y comparación ventajosa, contra otras personas, en relación con el segundo grupo de delincuentes. De acuerdo con los autores, a los narcotraficantes, por la misma naturaliza del delito, les es más fácil minimizar su conducta porque consideran que hay delitos más graves; además, tienden a deshumanizar, justamente porque no victimizan a personas específicas como ocurre en el grupo de delincuentes que han atentado contra otras personas (Giulio et al., 2018).

De otro lado, se ha identificado que experiencias tempranas de socialización parental orientadas al maltrato físico y psicológico, abuso y negligencia (Casey et al., 2017) son fuertes predictores de la agresividad, la falta de empatía y la conducta antisocial en la infancia y la adolescencia. El estudio de Campaert et al. (2018) evidencia que "en la infancia tardía, el castigo corporal repetido se asoció con niveles más altos de desvinculación moral un año después, y esta relación fue mediada por un aumento en la aprobación de agresión por parte de los padres" (p. 2656). Hyde et al. (2010) encontraron que la desconexión moral estaba relacionada con el rechazo parental a temprana edad y vivir en vecindarios muy vulnerables a nivel psicosocial.

Para Moore (2015), la desconexión moral es una orientación cognitiva relativamente estable, pero flexible a la influencia del contexto sociocultural, y que puede ser activada por más factores contextuales inmediatos (crianza, socialización con pares, modelamiento social, entre otros). Sin embargo, otras investigaciones (Arsenio \& Lemerise, 2004; Caprara, 1996) reportan que la influencia que la desconexión moral ejerce sobre el compromiso en los episodios agresivos y violentos para los adolescentes, llama la atención sobre los procesos psicológicos y modelos sociales que proveen el marco cognitivo dentro del cual la retaliación, la revancha y la violencia parecen ser apropiados y legítimos.

Estos hallazgos muestran cómo la desconexión moral es un factor importante de legitimación de la agresividad, la intimidación y la actividad delictiva, a la vez que se convierte en un inhibidor de la prosocialidad y la empatía (Muratori et al., 2017; Williford et al., 2015; Kokkinos \& Kipritsi, 2017). El estudio realizado por Muratori et al. (2017) muestra justamente que a mayor desconexión moral menor es 
el nivel de sensibilidad por el bienestar de los demás, lo cual evidencia que la empatía es un mediador psicológico entre la conducta prosocial y antisocial. La desconexión moral promueve la aparición de distintas conductas inaceptables socialmente y, por consiguiente, reducen la conducta prosocial y la empatía por el otro (Bandura 1999; Bandura et al., 1996; Van Noorden, Haselager, Cillessen \& Bukowski, 2014). Por tal motivo, tanto la empatía como la prosocialidad son factores negativamente asociado a la desconexión moral.

Tanto la conducta prosocial como la empatía se han considerado constructos multidimensionales en los que convergen factores motivacionales (Carlo \& Randall, 2002; Carlo, Hausmann, Christiansen \& Randall, 2003), cognitivos y afectivos (Davis, 1980, 1983). El primer término, hace referencia a todo comportamiento social positivo que tiene la intención de beneficiar a los demás (Aunè y Attorressi, 2017). Está asociado a la ayuda, la cooperación y el altruismo en las relaciones afectivas y en el cumplimiento de las normas sociales (Caprara \& Pastorelli, 1993; Gómez, 2018, 2019; Gómez \& Narváez, 2018).

Se ha considerado de manera consistente que factores psicosociales como la autoeficacia emocional, las emociones positivas, el autocontrol, la crianza y la parentalidad, el razonamiento moral prosocial, la empatía y la sociabilidad son predictores importantes de la prosocialidad en la infancia y la adolescencia (Richaud de Minzi \& Mesurado, 2016; Lemos \& Richaud de Minzi, 2010; Eisenberg, 1986; Martí Vilar, Iribarren Navarro, Grau-Martínez \& Olivera-La Rosa, 2018, Rodríguez, Mesurado, Ońate, Guerra \& Mengh, 2017; Gómez, 2019; Correa, 2017; Carlo, Crockett, Randall \& Roesch, 2007; Aguirre-Dávila, 2015; Auné, Blum, Abal, Lozzia \& Horacio, 2014); de tal modo que los procesos sociocognitivos, asociados al desarrollo de la prosocialidad, resultan ser antitéticos a la desconexión moral y la agresividad.

En cuanto a la empatía, diversos autores (Gutiérrez San Martín, Escartí \& Pascual, 2011; Richaud de Minzi, Lemos \& Mesurado, 2011; Richaud de Minzi \& Mesurado, 2016; Mestre, Samper \& Frías, 2002; Gómez, 2019) han considerado que es uno de los principales precursores de la prosocialidad y un predictor de la ayuda y la solidaridad en 
la infancia y la adolescencia. Esto debido a que involucra el reconocimiento del estado emocional de los demás, y una comprensión de su estado afectivo y psicológico (Richaud de Minzi, 2009). También se ha considerado que la empatía es un factor que favorece la disminución de la agresividad y la violencia en las personas. Ahora, el estudio realizado por Kokkino y Kipritsi (2017) en cuanto a la relación entre empatía y desconexión moral, muestra que los niños con baja empatía son más propensos a demostrar conductas orientadas a la desconexión moral y el acoso escolar.

Ahora bien, la investigación en torno a la relación entre prosocialidad, empatía y desconexión moral en adolescentes infractores de la ley es aún escasa, especialmente en población latinoamericana, lo cual plantea la importancia de ampliar la evidencia empírica en cuanto al estudio de los mecanismos de autorregulación y conducta social en esta población. Así como lo plantean Giulio et al. (2018) "los delincuentes son generalmente una población poco estudiada; de hecho, pocos estudios han examinado la separación moral de los delincuentes jóvenes y adultos, y en particular se ha prestado mucha menos atención a las diferencias entre los diferentes tipos de delincuentes" (p. 3).

En este sentido, este estudio ciertamente puede contribuir de manera significativa al desarrollo de estrategias de intervención y acompañamiento psicosocial en esta población, especialmente en lo que respecta a la estimulación de conductas sociales positivas en los procesos de re-educación y reintegración social. Dada la escasez de estudios que relacionan estos tres constructos psicológicos, a saber, la desconexión moral, la prosocialidad y la empatía, se considera que esta investigación puede tomarse como una contribución importante para ampliar los marcos teóricos sobre los factores asociados a los procesos cognitivosociales en jóvenes infractores en Latinoamérica y Colombia, en razón de que los patrones de pensamiento son un elemento fundamental para el desarrollo, mantenimiento y aumento de la actividad delictiva.

De acuerdo con lo expuesto hasta el momento, se planteó como objetivo identificar los mecanismos de desconexión moral y su relación con la empatía y la prosocialidad en adolescentes que han tenido 
conductas delictivas, en la ciudad de Manizales, Colombia. Se formuló la siguiente hipótesis: los diferentes mecanismos de desconexión moral en adolescentes infractores muestran una relación estadísticamente significativa, de signo negativo, con las conductas prosociales y la empatía; $y$, por el contrario, la prosocialidad y la empatía se relacionan de manera positiva.

\section{Método}

\section{Participantes}

Se contó con una muestra de 60 adolescentes $(50 \%$ hombres y $50 \%$ mujeres) de la ciudad de Manizales. Estos adolescentes incurrieron en actividades delictivas en los últimos dos años, tales como hurto, porte ilegal de armas, tráfico de estupefacientes e intentos de homicidio con arma cortopunzante. Las edades oscilan entre los 16 y los 19 ańos de edad. El 68.3\% ( $\mathrm{n}=41)$ entre 16 y 17 ańos, y el 31.7\% $(\mathrm{n}=19)$ entre los 18 y 19 ańos.

En cuanto a la tipología familiar, el $36.7 \%(n=22)$ pertenece a una familia monoparental materna, el $26.7 \%(n=16)$ a una familia extensa. El porcentaje restante $(n=22)$ que corresponde al $36.6 \%$ está distribuido de la siguiente manera: familias nucleares (13.3\%), viven con su pareja $(13.3 \%)$, familia mixta $(5 \%)$, monoparental paterno $(1.7 \%)$, vive con una hermana $(1.7 \%)$ y vive solo $(1.6 \%)$.

El 16.7\% $(n=10)$ de los adolescentes tienen un hijo, frente a un 83.3\% que no. En cuanto al grado de escolaridad se tiene que un 43.3 $\%$ está cursando la primaria, y un 56.7\% la básica media. El 71.7\% reporta consumo de sustancias psicoactivas de manera periódica, especialmente marihuana, cigarrillo, licor e inhalantes.

La aplicación de los instrumentos se hizo teniendo en cuenta las consideraciones éticas establecidas en la Ley 1090 de 2006, por medio de la cual se reglamenta el ejercicio de la profesión de Psicología y se dicta el Código Deontológico y Bioético, así como la Resolución 008430 de 1993 que establece las normas científicas, técnicas 
y administrativas para la investigación en salud. Esta investigación obedece a los principios éticos de respeto, intimidad y dignidad, asegurando la confidencialidad y el anonimato de los participantes, tal y como se establece en el artículo 26 y 50.

\section{Medición}

Para evaluar la desconexión moral se utilizó el Cuestionario de desconexión Moral - MMD (Bandura et al., 1996; Bandura, 2002) versión en español validada por Rubio-Garay, Amor y Carrasco (2017), que consta de 32 ítems tipo Likert de 5 opciones ( 1 = fuertemente en desacuerdo, hasta 5 = totalmente de acuerdo). Mide la inclinación a utilizar los diferentes mecanismos de desconexión moral establecidos por Bandura (2002): justificación moral $(\alpha=.91)$ ("Es correcto llegar a los golpes con el fin de proteger a los amigos"), lenguaje eufemístico $(\alpha=.81)$ ("Dar empujones es sólo una manera de bromear'), comparación ventajosa $(\alpha=.87)$ ("Robar un poco de dinero no es para nada grave si se piensa en las grandes sumas de dinero que otros se roban'), desplazamiento de la responsabilidad ( $\alpha=.89)$ ("Cuando los jóvenes viven en un barrio peligroso no se les puede reprochar el que sean agresivos"), difusión de la responsabilidad $(\alpha=.79)$ ("No se puede culpar a un miembro de una banda por los daños que causa la pandilla"), distorsión de las consecuencias $(\alpha=.89)$ ("Decir pequeñas mentiras no es tan grave, puesto que no hacen daño a nadie"), atribución de la culpa ( $\alpha=.78$ ) ("La gente que descuida sus cosas tiene la culpa si se las roban”) y deshumanización de la víctima $(\alpha=.91)$ ("Algunas personas merecen ser tratadas como animales"). Para el presente estudio se obtuvo un alfa de Cronbach de .94 para el instrumento general. La fiabilidad por subescalas osciló entre .78 a .91 tal y como se observa en la descripción de los mecanismos de desconexión moral.

Para estimar tanto el aspecto cognitivo como la reacción emocional del individuo al adoptar una actitud empática se utilizó el Índice de Reactividad Interpersonal - IRI (Davis, 1980, 1983) el cual consta de dos dimensiones y cuatro componentes de la empatía (Retuerto, 2004). La dimensión cognitiva se compone de la toma de perspectiva ("A menudo encuentro dificultades en ver las cosas desde el punto de vista 
de otra persona"), la cual hace referencia a la capacidad para adoptar la perspectiva de la otra persona, y la fantasía ("Sueño y fantaseo, bastante a menudo, acerca de las cosas que me podrian suceder"), que muestra la tendencia a establecer procesos de identificación con personas ficticias, sean del cine o la literatura. La dimensión afectiva está compuesta por la preocupación empática ("A menudo tengo sentimientos tiernos y de preocupación hacia la gente menos afortunada que yo"), la cual hace referencia a los sentimientos experimentados ante la experiencia negativa de otra persona, y el malestar personal ("En situaciones de emergencia me siento aprensivola e incómodola"), que evalúa la reacción emocional, por ejemplo, ansiedad, preocupación, estrés o tristeza, que se experimenta ante la exposición a situaciones o eventos negativos que vive otra persona. El índice de fiabilidad con alfa de Cronbach obtenido en este estudio fue de .73 , y en las escalas toma de perspectiva $(\alpha=.71)$, fantasía $(\alpha=.69)$, preocupación empática $(\alpha=.82)$ y malestar personal $(\alpha=.73)$ osciló entre .69 y .82

Las diferentes tendencias prosociales en la adolescencia fueron evaluadas con la Escala de tendencias prosociales-revisada- PTM-R (Carlo \& Randall, 2002) compuesta de 17 ítems que miden 6 tipos de tendencias o motivaciones prosociales: comportamiento prosocial público ("Puedo ayudar mejor a los otros cuando hay personas que están mirando"), comportamiento prosocial anónimo ("La mayoría de las veces ayudo a otros sólo si no saben quién les ha ayudado"), comportamiento prosocial emergencia (" $A$ menudo ayudo a los demás cuando están de verdad en problemas"), comportamiento prosocial emocional ("Me hace sentir bien cuando puedo dar consuelo a alguien que tiene problemas"), comportamiento prosocial por obediencia o complacencia ("Cuando los demás me piden ayuda no dudo en dárselas") y comportamiento prosocial altruista ("Creo que donar bienes materiales o dinero conviene más si recibo alguna ventaja” $(R)$ ). El índice de fiabilidad con alfa de Cronbach obtenido en este estudio fue de .81. La fiabilidad por subescalas osciló entre .68 y .86 . 


\section{Procedimiento}

Después de obtener la autorización del ICBF para llevar a cabo la investigación, se contactó la institución operadora del servicio de atención para el trabajo de campo. Los investigadores explicaron a los jóvenes el objetivo de la investigación, les presentaron el asentimiento informado, así como las características de las escalas a aplicar. Todos respondieron las escalas y, en los casos que lo requirieron, contaron con la asesoría de los investigadores allí presentes. La aplicación de los instrumentos tuvo una duración de 40 minutos aproximadamente.

\section{Análisis de datos}

Para el análisis se utilizó el paquete estadístico SPSS versión 22.0. Se realizó inicialmente un análisis de fiabilidad con alfa de Cronbach, seguido de un análisis descriptivo univariado de medias y desviaciones estándar de los instrumentos de acuerdo con las escalas y subescalas. Posteriormente se realizó un análisis de normalidad mediante la prueba Kolmogorov-Smirnov con el fin de determinar si las variables tienen o no una distribución normal. Una vez hecho esto, se realizó un análisis comparativo por género utilizando la prueba $\mathrm{T}$ de Student para muestras independientes en aquellos casos en que las variables presentaban una distribución normal, y la prueba no paramétrica $U$ de Mann-Whitney en los casos que no. Finalmente, se efectuó un análisis de correlación entre las variables de estudio: desconexión moral, tendencias prosociales y empatía.

\section{Resultados}

\section{Mecanismos de desconexión moral}

En la Tabla 1 se presentan los resultados descriptivos de los diferentes mecanismos de desconexión moral en los adolescentes estudiados, evidenciando que la dimensión que alcanza mayor valoración media es la de justificación moral $(M=2.60 ; D E=.95)$, lo cual indica 
que las acciones consideradas incorrectas por la sociedad, o que generan algún daño a los demás, se justifican a nivel cognitivo como un medio para lograr un fin moralmente correcto. El mecanismo que presenta la menor medida es la comparación ventajosa $(M=2.02 ; D E=1.02)$.

\section{Tabla 1}

Estadisticos descriptivos para la subescala Desconexión Moral

\begin{tabular}{lcc}
\hline Variables & $M$ & $D E$ \\
\hline Justificación moral & 2.60 & .95 \\
Lenguaje eufemístico & 2.21 & .85 \\
Desplazamiento de la responsabilidad & 2.24 & .95 \\
Difusión de la responsabilidad & 2.23 & .88 \\
Distorsión de las consecuencias & 2.27 & .79 \\
Atribución de la culpa & 2.23 & .98 \\
Deshumanización & 2.05 & .88 \\
Comparación ventajosa & 2.02 & 1.02 \\
\hline
\end{tabular}

\section{Conductas prosociales y empatía}

Para el caso de las conductas prosociales (Tabla 2), la tendencia prosocial por obediencia obtuvo el puntaje promedio más alto $(M=3.68 ; D E=1.01)$, seguido de la tendencia prosocial emergencia $(M=3.44 ; D E=1.04)$. Estos datos indican que la tendencia a ayudar a una persona por complacer y obedecer a los demás o en una situación de riesgo vital es mucho mayor a las conductas de ayuda mediadas por motivaciones altruistas o emocionales. Las medidas muestran que hay una menor tendencia a la ayuda en contextos públicos o que involucra presencia de muchas personas. En cuanto a la empatía, el mayor valor promedio se obtuvo en la dimensión de preocupación empática $(M=3.23$; $D E=.57)$; sin embargo, el malestar personal obtuvo el puntaje más bajo $(M=2.67 ; D E=.57)$. 


\section{Tabla 2}

Conductas prosociales y dimensiones de la empatía en las adolescentes infractoras

\begin{tabular}{lrrlrlrl}
\hline Tendencias prosociales & $M$ & $D E$ & $\begin{array}{l}\text { Dimensiones de la } \\
\text { empatía }\end{array}$ & $M$ & $D E$ \\
\hline Conducta prosocial público & 2.43 & 1.25 & Toma de perspectiva & 3.14 & .60 \\
Conducta prosocial anónimo & 2.56 & 1.07 & Fantasía & 2.92 & .70 \\
Conducta prosocial emergencia & 2.73 & .94 & Preocupación empática & 3.23 & .57 \\
Conducta prosocial emocional & 3.44 & 1.04 & Malestar personal & 2.67 & .57 \\
Conducta prosocial & 3.36 & 1.02 & & & & \\
complacencia & & & & & & \\
Conducta prosocial altruista & 3.06 & .67 & & & & \\
\hline
\end{tabular}

Se hizo el análisis comparativo de los mecanismos de desconexión moral, las dimensiones de la empatía y las tendencias prosociales en función del género (Tabla 3). Dado que no todas las variables presentaron una distribución normal de acuerdo con la prueba de KolmogorovSmirnov, se utilizó la Prueba U de Mann-Whitney; y para las variables con distribución normal, la $t$ de student. Se identifica que el género masculino presenta promedios más altos en todos los mecanismos de desconexión moral, y la diferencia es estadísticamente significativa $(p<.05)$. En cuanto a la empatía y las tendencias prosociales, la diferencia es inversa, el género femenino presenta promedios estadísticamente significativos $(p<.05)$ más altos. Se calculó el tamaño del efecto mediante la $d$ de Cohen, la cual arrojó puntuaciones superiores a .5, lo cual evidencia que las diferencias estadísticas encontradas son independientes del tamaño del efecto.

En cuanto al análisis comparativo de las variables de estudio, en función de la reincidencia en actividades delictivas, grado escolar y consumo de spa, no se encontraron diferencias estadísticamente significativas $(p<.05)$. Es de aclarar que la reincidencia se entiende como la repetición en más de una ocasión de una o más conductas criminales por parte de los adolescentes. 


\section{Tabla 3}

Análisis comparativo de los mecanismos de desconexión moral, las dimensiones de la empatía y las tendencias prosociales en función del género

\begin{tabular}{|c|c|c|c|c|c|c|c|}
\hline \multirow[t]{2}{*}{ Variables } & \multicolumn{2}{|c|}{$\begin{array}{c}\text { Femenino } \\
(\mathrm{n}=30)\end{array}$} & \multicolumn{2}{|c|}{$\begin{array}{l}\text { Masculino } \\
(\mathrm{n}=30)\end{array}$} & \multicolumn{3}{|c|}{ Estadísticos de prueba } \\
\hline & $M$ & $D E$ & $M$ & $D E$ & $Z$ & $t$ & $d$ \\
\hline Justificación moral & 2.27 & .92 & 2.94 & .87 & $-2.914^{* *}$ & & .75 \\
\hline Lenguaje eufemístico & 1.63 & .50 & 2.80 & .71 & $-5.337^{* * *}$ & & 1.91 \\
\hline $\begin{array}{l}\text { Desplazamiento de la } \\
\text { responsabilidad }\end{array}$ & 1.73 & .87 & 2.74 & .74 & & $-4.843^{* * *}$ & 1.25 \\
\hline $\begin{array}{l}\text { Difusión de la } \\
\text { responsabilidad }\end{array}$ & 1.69 & .68 & 2.76 & .73 & & $-5.853^{* * *}$ & 1.52 \\
\hline $\begin{array}{l}\text { Distorsión de las } \\
\text { consecuencias }\end{array}$ & 1.75 & .49 & 2.79 & .69 & $-5.314^{* * *}$ & & 1.74 \\
\hline Atribución de la culpa & 1.53 & .58 & 2.93 & .79 & $-5.611^{* * *}$ & & 2.02 \\
\hline Deshumanización & 1.37 & .44 & 2.73 & .64 & $-6.095^{* * *}$ & & 2.48 \\
\hline Comparación ventajosa & 1.28 & .49 & 2.76 & .85 & $-5.511^{* * *}$ & & 2.13 \\
\hline Prosocial público & 2.43 & 1.25 & 2.69 & .85 & -1.151 & & .24 \\
\hline Prosocial anónimo & 2.93 & 1.00 & 2.53 & .85 & $-1.978^{*}$ & & .43 \\
\hline Prosocial emergencia & 4.09 & .91 & 2.80 & .74 & & $6.053^{* * *}$ & 1.55 \\
\hline Prosocial emocional & 3.92 & .83 & 2.80 & .89 & $-4.288^{* * *}$ & & 1.30 \\
\hline Prosocial complacencia & 4.08 & .91 & 3.28 & .95 & $-3.037^{* *}$ & & .86 \\
\hline Prosocial altruista & 3.37 & .52 & 2.75 & .67 & $-3.684^{* * *}$ & & 1.03 \\
\hline Toma de perspectiva & 3.40 & .54 & 2.88 & .54 & & $3.74^{* * *}$ & .96 \\
\hline Fantasía & 3.23 & .76 & 2.61 & .47 & $-3.217^{* *}$ & & .98 \\
\hline Preocupación empática & 3.52 & .55 & 2.95 & .45 & & $4.422^{* * *}$ & 1.13 \\
\hline Malestar personal & 2.66 & .67 & 2.68 & .46 & & -.129 & .04 \\
\hline
\end{tabular}




\section{Correlaciones entre los mecanismos de desconexión moral, conductas prosociales y empatía}

Para verificar si se cumplen los supuestos de normalidad en cada una de las variables, se aplicó la prueba de Kolmogorov-Smirnov y se comprobó que no todas se distribuyen normalmente. Por tanto, se establecieron las correlaciones entre las variables calculando el coeficiente Rho de Spearman. En la Tabla 4 se observan correlaciones significativas $(p<.05)$ de signo negativo entre las diferentes las variables de estudio. En términos generales se puede evidenciar que los diferentes mecanismos de desconexión moral se relacionan de manera negativa con las conductas prosociales. La tendencia prosocial anónima presentó correlaciones negativas fuertes con los mecanismos de distorsión de las consecuencias, atribución de la culpa y comparación ventajosa. Las tendencias prosociales emocional, emergencia, complacencia y altruismo presentaron correlaciones negativas estadísticamente significativas $(p<.05)$ con todos los mecanismos de desconexión moral evaluados.

\section{Tabla 4}

Correlaciones entre los mecanismos de desconexión moral y las conductas prosociales

\begin{tabular}{lcccccccc}
\hline Variables & JM & LE & DR & DifR & DC & AC & Des & CV \\
\hline CCP & .05 & .04 & .01 & -.02 & -.03 & .16 & .06 & .11 \\
CPA & -.03 & -.21 & -.16 & -.24 & $-.33^{* *}$ & $-.30^{*}$ & -.23 & $-.34^{* *}$ \\
CPEm & $-.45^{* *}$ & $-.57^{* *}$ & $-.45^{* *}$ & $-.49^{* *}$ & $-.54^{* *}$ & $-.56^{* *}$ & $-.62^{* *}$ & $-.62^{* *}$ \\
CPE & $-.34^{* *}$ & $-.55^{* *}$ & $-.36^{* *}$ & $-.43^{* *}$ & $-.42^{* *}$ & $-.46^{* *}$ & $-.54^{* *}$ & $-.50^{* *}$ \\
CPC & $-.40^{* *}$ & $-.43^{* *}$ & $-.40^{* *}$ & $-.41^{* *}$ & $-.49^{* *}$ & $-.47^{* *}$ & $-.31^{*}$ & $-.52^{* *}$ \\
CPAlt & $-.42^{* *}$ & $-.40^{* *}$ & $-.36^{* *}$ & $-.30^{*}$ & $-.31^{*}$ & $-.49^{* *}$ & $-.40^{* *}$ & $-.59^{* *}$ \\
\hline
\end{tabular}

Nota: ${ }^{* *} p<.01,{ }^{*} p<.05$. JM=Justificación moral; LE=Lenguaje eufemístico; DR=Desplazamiento de la responsabilidad; DifR=Difusión de la responsabilidad; $\mathrm{DC}=$ Distorsión de las consecuencias; $\mathrm{AC}=$ Atribución de la culpa; Des=Deshumanización; $\mathrm{CV}=$ Comparación ventajosa; $\mathrm{CPP}=$ Comportamiento prosocial público; $\mathrm{CPA}=$ Comportamiento prosocial anónimo; $\mathrm{CPEm}=$ comportamiento prosocial emergencia; $\mathrm{CPE}=$ Comportamiento prosocial emocional; $\mathrm{CPC}=$ comportamiento prosocial complacencia; CPAlt= comportamiento prosocial altruista. 
En cuanto a la desconexión moral y su relación con la empatía (Tabla 5) se identificaron correlaciones negativas estadísticamente significativas $(p<.01)$ entre la toma de perspectiva y la preocupación empática con los diferentes mecanismos de desconexión moral. La dimensión de fantasía se correlaciona negativamente con la justificación moral $(r h o=-.36)$, lenguaje eufemístico $(r h o=-.42)$, distorsión de las consecuencias $(r h o=-.28)$, deshumanización $(r h o=-.46)$ y comparación ventajosa $(r h o=-.33)$. La dimensión de malestar personal no muestra correlaciones significativas.

\section{Tabla 5}

Correlaciones entre los mecanismos de desconexión moral y las dimensiones de la empatía

\begin{tabular}{lcccccccc}
\hline & JM & LE & DR & DifR & DC & AC & Des & CV \\
\hline TP & $-.45^{* *}$ & $-.52^{* *}$ & $-.30^{*}$ & $-.31^{*}$ & $-.42^{* *}$ & $-.48^{* *}$ & $-.47^{* *}$ & $-.53^{* *}$ \\
Fa & $-.36^{* *}$ & $-.42^{* *}$ & -.19 & -.24 & $-.28^{*}$ & -.20 & $-.46^{* *}$ & $-.33^{* *}$ \\
PE & $-.49^{* *}$ & $-.46^{* *}$ & $-.50^{* *}$ & $-.41^{* *}$ & $-.47^{* *}$ & $-.55^{* *}$ & $-.42^{* *}$ & $-.59^{* *}$ \\
$\mathrm{MP}$ & .23 & -.01 & .19 & .05 & .01 & .16 & -.05 & .17 \\
\hline
\end{tabular}

Nota: ${ }^{* *} p<.01,{ }^{*} p<.05$. JM=Justificación moral; LE=Lenguaje eufemístico; DR=Desplazamiento de la responsabilidad; DifR=Difusión de la responsabilidad; DC= Distorsión de las consecuencias; $\mathrm{AC}=$ Atribución de la culpa; Des=Deshumanización; $\mathrm{CV}=$ Comparación ventajosa; $\mathrm{TP}=\mathrm{Toma}$ de perspectiva; $\mathrm{Fa}=$ Fantasía; $\mathrm{PE}=$ Preocupación empática; $\mathrm{MP}=$ Malestar personal.

Finalmente, se puede observar que la conducta prosocial pública se correlaciona de manera significativa con el malestar personal $(r h o=.36$; $p<.01)$; la conducta prosocial anónima con la fantasía $(r h o=.30 ; p<.05)$ (tabla 6). La tendencia a ayudar en situaciones de emergencia y la conducta prosocial emocional presentan correlaciones significativas $(p<.01)$ con toma de perspectiva, fantasía y preocupación empática. La conducta prosocial por obediencia o complacencia presenta correlaciones con la preocupación empática $(r h o=.34 ; p<.01)$ y de toma de perspectiva $(r h o=.44 ; p<.01)$. Finalmente, la tendencia altruista se correlaciona con la preocupación empática $(r h o=.39 ; p<.01)$ y el malestar personal $(r h o=.28 ; p<.05)$. En términos generales se evidencian correlaciones 
fuertes entre las conductas prosociales y la empatía, especialmente con las dimensiones cognitivas (TP; Fa) y la preocupación empática.

\section{Tabla 6}

Correlaciones entre las conductas prosociales y las dimensiones de la empatía

\begin{tabular}{lcccccc}
\hline & CCP & CPA & CPEm & CPE & CPC & CPAlt \\
\hline TP & .19 & .18 & $.58^{* *}$ & $.70^{* *}$ & $.44^{* *}$ & .25 \\
$\mathrm{Fa}$ & .01 & $.30^{*}$ & $.49^{* *}$ & $.49^{* *}$ & .23 & .25 \\
$\mathrm{PE}$ & -.14 & .21 & $.60^{* *}$ & $.49^{* *}$ & $.34^{* *}$ & $.39^{* *}$ \\
$\mathrm{MP}$ & $.36^{* *}$ & .24 & .03 & .14 & -.02 & $-.28^{*}$ \\
\hline
\end{tabular}

Nota: ${ }^{* *} p<.01,{ }^{*} p<.05 . \mathrm{CPP}=$ Comportamiento prosocial público; $\mathrm{CPA}=$ Comportamiento prosocial anónimo; $\mathrm{CPEm}=$ comportamiento prosocial emergencia; $\mathrm{CPE}=$ Comportamiento prosocial emocional; $\mathrm{CPC}=$ comportamiento prosocial complacencia; $\mathrm{CPAlt}=$ comportamiento prosocial altruista; $\mathrm{TP}=$ Toma de perspectiva; $\mathrm{Fa}=$ Fantasía; $\mathrm{PE}=$ Preocupación empática; $\mathrm{MP}=$ Malestar personal.

\section{Discusión}

El objetivo de este estudio fue identificar los diferentes mecanismos de desconexión moral y su relación con la empatía y la prosocialidad en adolescentes que han tenido conductas delictivas, en la ciudad de Manizales, Colombia. En cuanto a la desconexión moral se identificó que se relaciona de manera negativa con la empatía, especialmente con las dimensiones de toma de perspectiva y preocupación empática; de igual manera, la prosocialidad, específicamente las motivaciones prosociales de emergencia, emocional, por complacencia y altruismo se asocian negativamente con los diferentes mecanismos de desvinculación moral. Estos hallazgos son coherentes con diversos estudios (Kokkinos \& Kipritsi, 2012; Van Noorden et al., 2014; Gini, Albiero, Benelli \& Altoè, 2007; Ortega, Sánchez \& Menesini, 2002; Moreno y Fernández, 2011; Garaigordobil \& García, 2006; Muratori et al., 2017; Jolliffe \& Farrington, 2006) en tanto evidencian que la desconexión moral se asocia de manera negativa con la empatía, tanto en su dimensión afectiva como cognitiva, y de manera positiva con la agresividad. 
Al respecto, el estudio de Kokkinos y Kipritsi (2017), realizado con 301 adolescentes escolares, mostró que la empatía, tanto cognitiva como afectiva, se asoció negativamente con la desconexión moral y la intimidación a los demás. Lo encontrado en este estudio evidencia que los adolescentes infractores demuestran un comportamiento moral desconectado, resultado de déficit en los aspectos emocionales de la empatía, a la vez que es un factor que inhibe la prosocialidad (Gini et al., 2007; Frick $\&$ White 2008; Hyde et al., 2010). En este sentido, la empatía es un mediador entre la conducta agresiva y la antisocial en tanto que, a mayor prosocialidad y empatía menor es el uso de procesos cognitivos de desconexión moral para justificar altos violentos o moralmente incorrectos (Gómez, 2019; Kokkinos \& Kipritsi, 2017).

El estudio realizado por Kiriakidis (2008), con 152 delincuentes juveniles en custodia, muestra que esta población presenta puntuaciones significativamente más altas en comparación con una muestra comunitaria; de igual manera, el realizado por Carroll (2009), indica que a mayor nivel de desconexión moral mayor es la relación con actitudes orientadas a apoyar o efectuar conductas delictivas; también muestra que, bajos niveles de juicio moral son usuales en personas que pertenecen a alguna hermandad, y de este modo se puede modelar procesos sociocognitivos asociados con la desconexión moral. Estos hallazgos reiteran la idea que los procesos cognitivos asociados a este constructo psicológico y los sesgos socioemocionales, en términos de una baja empatía y motivación prosocial, son factores fundamentales para comprender la conducta delictiva.

De otro lado, el mecanismo de desconexión más utilizado por los adolescentes que han tenido experiencias delictivas es la justificación moral, lo cual muestra la tendencia a anteponer medios que atentan contra el bienestar de otra persona o la normatividad social, para lograr fines considerados por los adolescentes como correctos o justos. Actos como hurtar o atentar contra la vida de otra persona son justificados moralmente si hay fines considerados morales, por ejemplo, proteger un ser querido; o fines más individualistas y utilitaristas como sobrevivir, comer, comprar cosas, entre otros. Este mecanismo de desconexión 
moral parece operar como un inhibidor de la prosocialidad, especialmente de la tendencia prosocial mediada por factores emocionales y por complacencia.

De igual manera, la tendencia a deshumanizar al otro, cuando se atenta contra él, implica inhibir los componentes afectivos de la empatía, como lo son el malestar personal y la preocupación empática, lo cual muestra que a mayor deshumanización menor es la empatía y viceversa. De este modo, la capacidad para experimentar un estado emocional o comprender el punto de vista del otro es determinante a la hora de desconectarse moralmente ante una conducta delictiva. La autocensura por una conducta cruel puede anularse despojando a las personas de las cualidades humanas. Una vez deshumanizadas, ya no son vistas como personas con sentimientos, esperanzas y preocupaciones, sino como un objeto infrahumano o como animales, lo que facilita el maltrato (Bandura, 1999, 2002). Por el contrario, como lo plantean Redondo et al. (2013) las personas que se preocupan por los sentimientos de los demás no tienden a deshumanizarlos; generan sentimientos similares de malestar cuando el otro está en una situación negativa. Bandura (citado por Caprara et al., 2014) reafirma ambas posturas: "los pensamientos morales pueden ser usados por un individuo para evitar la agresión o para comprometerse con ella haciéndola aceptable al justificarla como deseable o moral" (p. 71).

En relación con lo anterior, se evidencia un vacío investigativo en cuanto a la caracterización de los diferentes mecanismos asociados a los perfiles delincuenciales o al tipo de delito, lo que se podría considerar un campo interesante por explorar. Si bien en este estudio se evidenció que son la justificación moral y la distorsión de las consecuencias los mecanismos con mayor promedio, es necesario establecer nuevos acercamientos investigativos en cuanto a la relación que tiene cada mecanismo con delitos específicos y experiencias previas relacionadas, por ejemplo, historial de consumo de sustancias, abuso, maltrato o crianza deficitaria, redes de apoyo social, familiar y comunitario, entre otros.

Al respecto, el estudio realizado por Petruccelli et al. (2017) con una muestra de 362 hombres, dividida en tres grupos, 268 personas sin 
historial delictivos (grupo control), un grupo de 42 delincuentes sexuales y 52 delincuentes no sexuales, muestran que los delincuentes sexuales hacen mayor uso de todos los mecanismos de desconexión moral en comparación a los delincuentes no sexuales; de igual manera, se evidenciaron diferencias estadísticamente significativas en justificación moral, atribución de la culpa, comparación ventajosa y deshumanización entre el grupo control y la población infractora (sexuales y no sexuales).En el mismo sentido, el estudio realizado por Giulio y Pace (2018) muestra que los narcotraficantes presentan mayor desconexión moral general, deshumanización y comparación ventajosa que los transgresores de otras personas.

Estos hallazgos reiteran la necesidad de establecer estudios mucho más sensibles en relación a los mecanismos específicos de desvinculación moral asociados a experiencias delictivas particulares y factores relacionados. De acuerdo con Casey et al. (2017), Muratori et al. (2017), Giulio \& Pace (2018), estas diferencias en cuanto a los mecanismos más prevalentes no se relacionan solamente con el tipo de delito, sino que también tienen que ver, de manera mucho más directa, con aspectos sociales y afectivos de los infractores, entre ellos, el rechazo temprano en la crianza, la vulnerabilidad psicosocial en el barrio o vecindario, déficit en empatía, violencia social, formas inapropiadas de castigo, abuso y negligencia en la crianza, consumo de drogas, entre otros, los cuales se deben considerar en próximos estudios. De otro lado, también se puede considerar que la desconexión moral es un constructo psicológico independiente de los factores de vulnerabilidad psicosocial del infractor, que debe estudiarse más en relación a los aspectos sociocognitivos y emocionales que contextuales. Al respecto, el estudio de Kiriakidis (2008) concluye justamente que:

[...] la falta de relación de la desconexión moral con la mayoría de las características sociales, familiares, escolares, laborales, legales y de estilo de vida de la muestra sugiere que la desconexión moral es una variable independiente que ejerce una influencia en el comportamiento del delincuente juvenil por encima de las características sociales. de delincuentes juveniles (p. 571). 
Lo cierto es que, para determinar con mayor precisión si la desconexión moral es un constructo dependiente o no de los factores contextuales y psicosociales del infractor, es necesario un mayor soporte empírico y nuevas aproximaciones investigativas en esta línea de trabajo.

De otro lado, las motivaciones prosociales en los adolescentes estudiados están dirigidas a la ayuda en situaciones de emergencia y para complacer u obedecer a otra persona, y no necesariamente por motivaciones altruistas o emocionales. De acuerdo con Batson y Powell (2003) la prosocialidad no debe entenderse solo en términos de motivaciones altruistas, dado que pueden darse conductas de ayuda mediadas por factores utilitaristas o motivaciones colectivistas, es decir, ayudar al otro para beneficio propio o a un grupo específico de personas, por ejemplo, amigos, familiares y otros. De este modo, pueden darse conductas prosociales que no son necesariamente altruistas (Martí Vilar \& Lorente 2010; Gómez, 2019). Una tendencia que también se evidencia es coherente con el estudio realizado por Gil (2016) donde se encontró que cuando hay más espectadores disminuye la probabilidad de ayuda al otro.

En cuanto a las variaciones encontradas con respecto al género, se observan algunos hallazgos interesantes. Los hombres presentaron medias más altas, estadísticamente significativas con un tamaño del efecto alto, en los mecanismos de desconexión moral, y menor prosocialidad y empatía en comparación con las mujeres, lo cual indica una mayor propensión en el género masculino a continuar asumiendo conductas delictivas a futuro e inhibir las conductas de ayuda o empatía ante las acciones que atentan contra el otro o las normas sociales. Estos resultados coinciden con lo reportado por De Caroli, Sagone y Falanga (2011) en su estudio realizado con estudiantes italianos, según el cual los hombres usaron la desconexión moral más que las mujeres (p. 111). Por el contrario, en el estudio realizado en Sevilla (España) y Florencia (Italia) por Ortega et al. (2002) encontraron similares niveles en el uso de mecanismos de desconexión moral en ambos sexos. Estudios similares en entornos educativo (Jolliffe \& Farrington, 2011; Navarro, Maluenda \& Varas, 2016) indican que las mujeres muestran una mayor 
empatía y tienen menor probabilidades de involucrarse en actividades de intimidación o acoso en comparación a los hombres. De igual manera, se ha encontrado que la relación entre desconexión moral y bullying es más fuerte en los hombres (Paciello et al., 2008)

De acuerdo con Mestre et al. (2002) las personas más inestables emocionalmente, con menos recursos para manejar la impulsividad, son las más propensas a la agresividad y, por tanto, en este caso, a la reincidencia en actividades delictivas. No obstante, el aumento de las conductas prosociales contribuye a disminuir la agresividad en adolescentes (Caprara et al., 2015).

De acuerdo con los resultados obtenidos en la presente investigación, se evidencia además una correlación negativa entre variables dependientes e independientes, lo cual indica que a mayor presencia de conductas prosociales y empatía por el otro, menor será el uso de mecanismos de justificación de actos considerados moralmente incorrectos. De esta manera se puede afirmar que, si las personas presentan más comportamientos prosociales vinculados con la ayuda al otro, disminuye la tendencia a justificar y comparar malas acciones o a asumir conductas violentas. Esta afirmación también se encuentra en el estudio de Tur (2003) en el que muestra que las personas con estabilidad emocional tienden a presentar conductas prosociales, y las personas inestables emocionalmente tienden a presentar conductas agresivas.

De igual manera se identifica que entre más alta sea la capacidad empática, tanto en sus dominios cognitivos como afectivos, menor es la tendencia a justificar acciones inmorales y violentas. En este sentido, la empatía funciona como factor protector ante la agresividad y la desconexión moral (Gómez, 2019; Correa, 2017; Samper, 2014; Richaud de Minzi \& Mesurado, 2016; Richaud de Minzi, 2009, 2014). Es de mencionar que el componente de malestar personal presentó el promedio más bajo y se relacionó de manera significativa con la desconexión moral. A pesar de que el componente afectivo de preocupación empática presentó el promedio más alto, el de malestar personal es el más bajo, lo que puede estar asociado a una baja capacidad para experimentar reacciones emocionales negativas ante la exposición 
a situaciones o eventos negativos que vive otra persona. De acuerdo con Gómez y Narváez $(2017,2018)$ cuando un adolescente ha sido expuesto de manera prolongada a eventos de violencia tiende a generar una respuesta desensibilizada ante la experiencia y las necesidades de los demás.

En coherencia con lo anterior, los hallazgos muestran que el componente de malestar personal se relaciona de manera negativa con la conducta altruista, y es la única variable que se correlaciona con la tendencia a ayudar en público, lo cual muestra que la tendencia a ayudar a otros en presencia de espectadores está mediada por motivaciones no altruistas; una de ellas es reducir el malestar personal.

Finalmente, lo encontrado en este estudio, en cuanto a las correlaciones positivas entre las conductas prosociales y los componentes de la empatía, es coherente con diversos estudios a nivel nacional e internacional (Garaigordobil \& Maganto, 2011; Richaud de Minzi \& Mesurado, 2016; Redondo, Rangel \& Luzardo, 2015; Mestre, Samper, Tur, Cortés \& Nácher, 2006; Mestre et al., 2002; Gómez, 2017, 2019; Gómez \& Narváez, 2017, 2018) que muestran que la empatía es un precursor de la conducta social positiva, y que tanto los componentes afectivos como cognitivos son determinantes para el desarrollo de la prosocialidad en la adolescencia. Cuando se da un comportamiento prosocial es porque la persona tiene la capacidad de ponerse en el lugar del otro, aspecto evidenciado en el estudio realizado por Garaigordobil \& García (2006) donde se ratifica que la empatía está relacionada de manera positiva con las conductas prosociales, aspecto que también fue evidenciado por Mestre et al. (2006) quienes concluyen que la emocionalidad regulada y la empatía se relacionan fuertemente con la conducta prosocial.

Los hallazgos encontrados en esta investigación y la discusión realizada, se pueden considerar de gran utilidad tanto para la investigación y la intervención psicosocial con esta población, especialmente, en los programas de atención especializada del sistema de responsabilidad penal para adolescentes y las políticas públicas en infancia y adolescencia en Colombia, y sus equivalentes para otros países en América 
Latina. Se considera que el estudio de la desconexión moral y los factores asociados tanto positiva como negativamente es un campo aún muy reciente y, por tal motivo, se considera que las repercusiones prácticas y teóricas de este estudio pueden ir orientadas a la comprensión de los factores sociocognitivos de la conducta delictiva, además de las posibilidades de abordaje de los mecanismos psicológicos que subyacen a la conducta antisocial, más allá de las miradas estigmatizantes o las simples descripciones asociadas al delito.

Lo anterior, evidentemente abre múltiples aristas en lo que respecta el abordaje y tratamiento, pues, así como lo manifiestan Hyde et al. (2010) "A largo plazo, la investigación sobre la DM y otros factores cognitivos involucrados en el comportamiento antisocial puede ayudar no solo con la planificación del tratamiento, sino que también podría ser útil para identificar subgrupos de niños con comportamiento antisocial" (p. 207). Añadido a esto, y como se mencionó al inicio, son escasos los estudios donde relacionan estos constructos psicológicos, en especial, la desconexión moral en población infractora, razón por la cual estos hallazgos se pueden tomar como un referente para ampliar los marcos teóricos sobre los factores asociados a los procesos cognitivosociales en jóvenes infractores en Colombia y Latinoamérica.

De igual manera, se resalta la importancia de generar programas y estrategias que estimulen la empatía, la solidaridad, la educación emocional y criterios morales prosociales en los procesos re-educativos y de reintegración a la vida social, pues se considera que la promoción de prácticas solidarias y la exposición a un modelamiento prosocial puede generar nuevas formas de aprendizaje social en las que el adolescente logre una mayor sensibilidad por el bienestar de los demás, lo cual puede incidir en la disminución a lo largo del tiempo de la desconexión moral, la reducción del delito y la reincidencia del mismo en la población joven.

Es importante, además, resaltar algunas limitaciones en este estudio. Primero, dado que el tamaño de la muestra fue pequeño, hay que tener cautela con la generalización de los resultados. También se resalta que no se tuvo un grupo control para realizar comparaciones en las mediciones, lo cual habría aportado mucha más información. 
Para futuros estudios, es importante determinar con mayor precisión las causas del cambio en la desconexión moral y factores psicológicos asociados en población infractora, posterior a procesos re-educativos y de tratamiento en centros especialización para esto, lo cual remite a la importancia de efectuar estudios longitudinales en los que la investigación y la intervención se articulen. Otro aspecto por explorar a futuro, y que resulta ser una limitación en este estudio, es la relación que presenta la desconexión moral y sus diferentes mecanismos con factores contextuales y psicosociales asociados, entre ellos, características sociales, familiares, psicológicas, escolares, laborales, legales y de estilo de vida de los infractores, con el fin de determinar la dependencia o independencia de este constructo psicológico con dichas características.

\section{Referencias}

Aguirre-Dávila, E. (2015). Prácticas de crianza, temperamento y comportamiento prosocial de estudiantes de educación básica. Revista Latinoamericana de Ciencias Sociales, Niñez y Juventud, 13(1), 223-243. https://doi.org/10.11600/1692715x.13113100314

Arsenio, W. \& Lemerise, E. (2004). Aggression and moral development: Integrating the social information processing and moral domain models. Child Development, 75, 987-1002. https://doi. org/10.1111/j.1467-8624.2004.00720.x

Auné, S. E., Blum, D., Abal, J. P., Lozzia, G. S. \& Horacio, F. A. (2014). La Conducta Prosocial: Estado actual de la investigación. Perspectivas en Psicología, 11(2), 21-33. Recuperado de http://www. redalyc.org/articulo.oa?id $=483547666003$

Auné, S. E. \& Attorresi, H. F. (2017). Dimensionalidad de un test de conducta prosocial. Revista Evaluar, 17(1), 29-37. Recuperado de https://revistas.unc.edu.ar/index.php/revaluar

Bandura, A. (1990). Selective Activation and Disengagement of Moral Control. Journal of Social Issues, 46(1), 27-46. https://doi.org/ 10.1111/j.1540-4560.1990.tb00270.x 
Bandura, A. (1991). Social Cognitive Theory of Moral Thought and Action. En W. M. Kuritines, \& J. L. Gewirtz (Eds.), Handbook of Moral Behavior and Development (Vol. 1, pp. 45-103). Hillsdale, NJ: Lawrence Erlbaum Associates.

Bandura, A. (1999). Moral disengagement in the perpetration of inhumanities. Personality and social Psychology Review, 3(3), 193209. https://doi.org/10.1207/s15327957pspr0303_3

Bandura, A. (2002). Selective moral disengagement in the exercise of moral agency. Journal of Moral Education, 31(2), 101-119. https://doi.org/10.1080/0305724022014322

Bandura, A. (1988). Mechanisms of moral disengagement. In W. Reich (Ed.), Origins of terrorism: Psychologies, ideologies, theologies, states of mind (pp. 161-191). Washington, D.C.: Woodrow Wilson Center Press.

Bandura, A., Underwood, B. \& Fromson, M. E. (1975). Disinhibition of aggression through diffusion of responsibility and dehumanization of victims. Journal of Research in Personality, 9, 253-269. https://doi.org/10.1016/0092-6566(75)90001-X

Bandura, A. (2004). The role of selective moral disengagement in terrorism and counterterrorism. En F. M. Moghaddam \& A.J. Marsella (Eds.), Understanding terrorism: Psychosocial roots, consequences, and interventions (pp. 121-150). Washington, D.C.: American Psychological Association. https://doi.org/ 10.1037/10621-006

Bandura, A., Barbaranelli, C., Caprara, G, V. \& Pastorelli, C. (1996). Mechanisms of Moral Disengagement in the Exercise of Moral Agency. Journal of Personality and Social Psychology, 71(2). 364374. https://doi.org/10.1037/0022-3514.71.2.364

Bandura, A., Caprara, G. V., Barbaranelli, C., Pastorelli, C. \& Regalia, C. (2001). Sociocognitive Self-Regulatory Mechanisms Governing. Transgressive Behavior, 80(1), 125-135. https://doi. org/10.1037//0022-3514.80.1.125

Batson, C. D. \& Powell, A. (2003). Altruism and prosocial behavior. En M. Theodore \& L. Melvin (Eds.), Handbook of Psychology: 
Personality and Social Psychology, (5). Nueva York: John Wiley y Sons, Inc. XIX. https://doi.org/10.1002/0471264385.wei0519 Boardleya, I.A., Smith, A.L., Mills, J., Grixa, j., Wynnea, C. \& Wilkins, L.(2018). Development of moral disengagement and self-regulatory efficacy assessments relevant to doping in sport and exercise. Psychology of Sport \& Exercise, 36, 57-70. https:// doi.org/10.1016/j.psychsport.2018.01.007

Bussey, K., Quinn, C. \& Dobson, J. (2015). The moderating role of empathic concern and perspective taking on the relationship between moral disengagement and aggression. Merrill- Palmer Quarterly,61(1),10-29.https://doi.org/10.13110/merrpalmquar 1982.61.1.0010

Campaert, K., Nocentini, A. \& Menesini, E. (2018). The Role of Poor Parenting and Parental Approval for Children's Moral Disengagement. Journal of Child and Family Studies, 27, 2656. https://doi.org/10.1007/s10826-018-1097-1

Casey, E. A., Masters, N. T., Beadnell, B., Hoppe, M. J., Morrison, D. M. \& Wells, E.A. (2017). Predicting sexual assault perpetration among heterosexually active young men. Violence Against Women, 23(1), 3-27. https://doi.org/10.1177\%2F1077801216634467

Caprara, G.V. (1996). Structures and processes in personality psychology. European Psychologist, 1, 14-26. https://doi.org/10. 1027/1016-9040.1.1.14

Caprara, G.V. \& Pastorelli, C. (1993). Early emotional instability, prosocial behaviour, and aggression: Some methodological aspects. European Journal of Personality, 7(1), 19-36. http://dx. doi.org/10.1002/per.2410070103

Caprara, G.V., Alessandri, G., Fida, R., Tisak, M., Fontaine R. \& Paciello, M. (2014). The contribution of moral disengagement in mediating individual tendencies toward aggression and violence. Developmental Psychology, 50(1), 71-85. https://doi.org/ 10.1037/a0034488

Caprara, G.V., Luengo, P. B., Zuffiano, A., Gerbino, M. \& Pastorelli, C. (2015). Why and How to Promote Adolescent's Prosocial 
Behaviors: Direct, Mediated and Moderated Effects of the Cepidea School-based Program. J. Youth Adolescence. https:// doi.org/10.1007/s10964-015-0293-1

Carlo, G. \& Randall, B.A. (2002). The development of a measure of prosocial behaviors for late adolescents. Journal of Youth and Adolescence 31, 31-44. https://doi.org/10.1023/ A: 1014033032440

Carlo, C., Hausmann, A., Christiansen, S. \& Randall, B. (2003). Sociocognitive and behavioral correlates of a measure of prosocial tendencies for adolescents. Journal of Early Adolescence 23(1), 107-134. https://doi.org/10.1177/0272431602239132

Carlo, G., Crockett, L. J., Randall, B.A. \& Roesch, S. C. (2007). A latent growth curve analysis of prosocial behavior among rural adolescents. Journal of Research on Adolescence, 17(2), 301-324. https://doi.org/10.1111/j.1532-7795.2007.00524.x

Carroll, J.A. (2009). Impact of Moral Judgment and Moral Disengagement on Rape Supportive Attitudes in College Males (Unpublished doctoral dissertation), University of Alabama Tuscaloosa. Recuperado de https://ir.ua.edu/bitstream/handle/ 123456789/623/file_1.pdf?sequence $=1$ \&isAllowed $=y$

Correa, M.C. (2017). Aproximaciones epistemológicas y conceptuales de la conducta prosocial. Revista Zona Próxima, 27(2), 1-21. http://dx.doi.org/10.14482/zp.27.10978

Davis, M.H. (1980). A multidimensional Approach to Individual Differences in Empathy. JSAS Catalog of Selected Documents in Psychology, 10, 85. Recuperado de https://www.uv.es/friasnav/ Davis_1980.pdf

Davis, M.H. (1983). Measuring Individual Differences in Empathy: Evidence for a Multidimensional Approach. Journal of Personality and Social Psychology, 44(1), 113-126. http://dx.doi. org/10.1037/0022-3514.44.1.113

DeLisi, M., Peters, D. J., Dansby, T., Vaughn, M.G., Shook, J.J. \& Hochstetler, A. (2014). Dynamics of psychopathy and moral disengagement in the etiology of crime. Youth Violence and 
Juvenile Justice, 12(4), 295-314. https://doi.org/10.1177\% 2F1541204013506919

De Caroli, M.E., Sagone, E. \& Falanga, R. (2011). Civic Moral Disengagement and Personality. A Comparison Between Law and Psychology Italian Students. International Journal of Developmental and Educational Psychology, 5(1), 105-112.

Doyle, F. L. \& Bussey, K. (2017). Moral disengagement and children's propensity to tell coached lies. Journal of Moral Education, 47(1), 91-103. https://doi.org/10.1080/03057240.2017.1380611

Eisenberg, N. (1986). Altruistic emotion, cognition and behavior. Hillsdale, NJ: Erlbaum.

Garaigordobil, M. \& García, P. (2006). Empatía en niños de 10 a 12 años. Psicothema, 18(2), 180-186. Recuperado de http://www. psicothema.com/pdf/3195.pdf

Frick, P. J. \& White, S. F. (2008). Research review: the importance of callous-unemotional traits for developmental models of aggressive and antisocial behavior. Journal of Child Psychology and Psychiatry, 49(4), 359-375. https://doi.org/10.1111/j.14697610.2007.01862.x

Garaigordobil, M. \& Maganto, C. (2011). Empatía y resolución de conflictos durante la infancia y la adolescencia. Revista Latinoamericana de Psicología, 43(2). 255-266. Recuperado de http://www.scielo.org.co/scielo.php?script=sci_arttext\&pid= S0120-05342011000200005

Navarro, G., Maluenda, J. \& Varas, M. (2016). Diferencias en empatía según sexo y área disciplinar en estudiantes universitarios chilenos de la provincia de Concepción, Chile. Educación, 25(49), 63-82. https://doi.org/10.18800/educacion.201602.004

Gil, T.L. (2016). Conductas prosociales y antisociales en adolescentes. Medellín: Facultad de Ciencias Sociales y Humanas de la Universidad de Antioquia.

Gini, G. (2006). Social Cognition and Moral Cognition in Bullying: What's Wrong?. Aggressive Behavior, 32, 528-539. https://doi. org/10.1002/ab.20153 
Gini, G., Pozzoli, T. \& Hymel, S. (2014). Moral Disengagement Among Children and Youth: a Meta-analytic Review of Links to Aggressive Behavior. Aggressive Behavior, 40(1), 56-68. https:// doi.org/10.1002/ab.21502

Gini, G., Pozzoli, T. \& Bussey, K. (2015). Moral disengagement moderates the link between psychopathic traits and aggressive behavior among early adolescents. Merrill-Palmer Quarterly, 61(1), 51-67. https://doi.org/10.13110/merrpalmquar1982.61.1.0051

Gini, G., Pozzoli, T. \& Hauser, M. (2011). Bullies have enhanced moral competence to judge relative to victims, but lack moral compassion. Personality and Individual Differences, 50, 603-608. https://doi.org/10.1016/j.paid.2010.12.002

Gini, G., Albiero, P., Benelli, B. \& Altoè, G. (2007). Does empathy predict adolescents' bullying and defending behavior? Aggressive Behavior, 33, 467-476. https://doi.org/10.1002/ab.20204

Gini, G., Pozzoli, T. \& Bussey, K. (2015). Moral disengagement moderates the link between psychopathic traits and aggressive behavior among early adolescents. Merrill-Palmer Quarterly, 61(1), 51-67. https://doi.org/10.13110/merrpalmquar1982.61. 1.0051

Gini, G., Pozzoli, T. \& Bussey, K. (2014). Collective moral disengagement: Initial validation of a scale for adolescents. European Journal of Developmental Psychology, 11, 386-395. https://doi.org/10.1080/17405629.2013.851024

Giulio, D., Petruccelli, I. \& Pace, U. (2018). Drug use as a risk factor of moral disengagement: A study on drug traffickers and offenders against other persons. Psychiatry, Psychology and Law. Advance online publication. https://doi.org/10.1080/13218719.2018.14 37092

Gómez, A.S. y Narváez, N. (2018). Prosocialidad en niños, niñas y adolescentes desvinculados de grupos armados ilegales. retos y reflexiones para la investigación social. Diversitas. Perspectivas en Psicología, 14(2), 263-278. https://doi.org/10.15332/s17949998.2018 .0014 .05 
Gómez, A.S. (2019). Prosocialidad. Estado actual de la investigación en Colombia. Revista Colombiana de Ciencias Sociales, 10(1), 1-42. Recuperado de http://www.funlam.edu.co/revistas/index. php/RCCS/article/view/3065/2300

Gómez, A. (2018). De la cosmología peirceana a la evolución social. Reflexiones sobre el agapismo y los hábitos sociales en sentido evolutivo. Cuadernos de Filosofía Latinoamericana, 39(118), 37-58. https://doi.org/10.15332/10.15332/s0120-8462.2018. 0118.02 .

Gómez, A. S. y Narváez, N. (2017). Conductas prosociales en adolescentes desvinculados de grupos armados ilegales, pertenecientes a la modalidad Hogar Sustituto Tutor del ICBF, en Manizales. En V., Castellanos (comp). Innovaciones en Psicología y Salud Mental. Ponencia presentada en el IV Congreso Internacional y IX Nacional en Tunja.

Gómez, A. S. (2017). Prosocialidad: una propuesta alternativa para el trabajo con nińos, nińas y adolescentes desvinculados de grupos armados ilegales. En S. A. Alvarado. y P. A, Vommaro (Comps.), Infancias y juventudes iberoamericanas: transformaciones democráticas, justicia social y procesos de construcción de paz. Centro de Estudios Avanzados en Niñez y Juventud - Fundación Centro Internacional de Educación y Desarrollo Humano, CINDE - Universidad de Manizales - CLACSO. Centro Editorial CINDE.

Gutzwiller-Helfenfinger, E. (2015). Moral disengagement and aggression: Comments on the special issue. Merrill-Palmer Quarterly, 61(1), 192-211. https://doi.org/10.13110/ merrpalmquar1982.61.1.0192

Gutiérrez San Martín, M., Escartí, A. \& Pascual, C. (2011). Relaciones entre empatía, conducta prosocial, agresividad, autoeficacia y responsabilidad personal y social de los escolares. Psicothema, 23(1), 13-19. Recuperado de http://www.psicothema.com/pdf/ 3843.pdf 
Hardy, A. M., Bean, D. S \& Olsen, J.A. (2014). Moral Identity and Adolescent Prosocial and Antisocial Behaviors: Interactions with Moral Disengagement and Self-regulation. J Youth Adolescence, https://doi.org/10.1007/s10964-014-0172-1

Hymel, S. \& Perren, S. (2015). Introduction to the special issue: Moral disengagement and aggression in children and youth. MerrillPalmer Quarterly, 61(1), 1-9. https://dx.doi.org/10.13110/ merrpalmquar1982.61.1.0001

Hyde, L. W., Shaw, D. S. \& Moilanen, K. L. (2010). Developmental precursors of moral disengagement and the role of moral disengagement in the development of antisocial behavior. Journal of Abnormal Child Psychology, 38(2), 197-209. https:// doi.org/10.1007/s10802-009-9358-5.

Houwing, F. L. \& Bussey, K. (2017). Moral disengagement and the propensity to endorse physical punishment practices. Journal of Child and Family Studies, 26, 1206-1218. https://doi. org/10.1007/s10826-016-0643-y

Jolliffe, D. \& Farrington, D.P. (2006). Examining the relationship between low empathy and bullying. Aggressive Behavior, 32, 540-550. https://doi.org/10.1002/ab.20154

Jolliffe, D. \& Farrington, D. P. (2011). Is low empathy related to bullying after controlling for individual and social background variables? Journal of Adolescence, 34, 59-71. https://doi. org/10.1016/j.adolescence.2010.02.001

Kiriakidis, S. P. (2008). Moral Disengagement: Relation to Delinquency and Independence From Indices of Social Dysfunction. International Journal of Offender Therapy and Comparative Criminology, 52(5), 571-583. https://doi.org/10.1177/0306624 X07309063

Kokkinos, C. M. \& Kipritsi, E. (2012). The relationship between bullying, victimization, trait emotional intelligence, self-efficacy and empathy among preadolescents. Social Psychology of Education, 15, 41-58. https://doi.org/10.1007/s11218-011-9168-9 
Kokkinos, C. M. \& Kipritsi, E. (2017). Bullying, moral disengagement and empathy: exploring the links among early adolescents. Educational Psychology, 1-18. https://doi.org/10.1080/0144341 0.2017 .1363376

Lemos, V. N. \& Richaud de Minzi, M. C. (2010). Construcción de un instrumento para evaluar el razonamiento prosocial en nińos de 7 y 8 años: una versión pictórica. Universitas Psychologica, 9(3), 879-891. https://doi.org/10.11144/Javeriana.upsy9-3.cier

Lucidi, F., Zelli, A., Mallia, L., Grano, C., Russo, P. M. \& Violani, C. (2008). The social-cognitive mechanisms regulating adolescents' use of doping substances. Journal of Sports Sciences, 26, 447-456. https://doi.org/10.1080/02640410701579370

Martí Vilar, M., Iribarren Navarro, M. V., Grau-Martínez, L. \& Olivera-La Rosa, A. (2018). Actitudes religiosas, valores y razonamiento moral prosocial en una muestra adolescente. Revista Colombiana de Ciencias Sociales, 9(1), 155-175. https:// doi.org/10.21501/22161201.2613

Martí Vilar, M. \& Lorente, S. (2010). Factores determinantes de las conductas prosociales. En M. Martí Vilar, Razonamiento Moraly Prosocialidad. Fundamentos. Madrid: Ccs.

Martínez-Morales, M. B., Robles-Haydar, C. A., Amar-Amar, J. J. \& Crespo-Romero, F. A. (2016). Crianza y desconexión moral en infantes: su relación en una comunidad vulnerable de Barranquilla. Revista Latinoamericana de Ciencias Sociales, Niñezy Juventud, 14(1), 315-330. https://doi.org/10.11600/1692715x. 14121011214

Mestre, E. M., Samper, G. P. \& Frías, N. M. (2002). Procesos cognitivos y emocionales predictores de la conducta prosocial y agresiva: La empatía como factor modulador. Psicothema, 14(2), 227-232. Recuperado de http://www.psicothema.com/pdf/713.pdf

Mestre, E. M., Samper, G. P., Tur, M. A., Cortés. T. \& Nácher, M. J. (2006). Conducta prosocial y procesos psicológicos implicados: un estudio longitudinal en la adolescencia. Revista Mexicana de Psicología, 23(2), 203-215. Recuperado de http://www.redalyc. org/pdf/2430/243020649006.pdf 
Moore, C. (2015). Moral Disengagement. Current Opinion in Psychology, 6, 199-204. https://doi.org/10.1016/j.copsyc.2015.07.018

Muratori, P., Paciello, M., Buonanno, C., Milone, A., Ruglioni, L., Lochman, J.E. \& Masi, G. (2017). Moral disengagement and callous-unemotional traits: A longitudinal study of Italian adolescents with a disruptive behavior disorder. Criminal Behaviour and Mental Health, 27(5), 514-524. https://doi.org/10.1002/ cbm.2019

Moreno, J.E. \& Fernández, C. (2011). Empatía y flexibilidad yoica, Su relación con la agresividad y la prosocialidad. Limite, 6(23), 41-55. Recuperado de http://www.redalyc.org/pdf/836/83620927004. pdf

Ortega, R.R., Sánchez, V. \& Menesini, E. (2002). Violencia entre iguales y desconexión moral: un análisis transcultural. Psicothema, 14(1), 37-49. Recuperado de https://idus.us.es/ xmlui/bitstream/handle/11441/56454/Violencia\%20entre\%20 iguales.pdf? sequence $=1$ \&isAllowed $=y$

Osofsky, M. J., Bandura, A. \&Zimbardo, P. G. (2005). The role of moral disengagement in the execution process. Law and Human Behavior, 29, 371-393. https://doi.org/10.1007/s10979-005-4930-1

Paciello, M., Fida, R., Tramontano, C., Lupinetti, C. \& Caprara, G. V., (2008). Stability and change of moral disengagement and its impact on aggression and violence in late adolescence. Child Development, 79(5), 1288-1309. https://doi.org/10.1111/ j.1467-8624.2008.01189.x

Petruccelli, I., Simonelli, C., Barbaranelli, C., Grilli, S., Tripodi, M. F. \& D’Urso, G. (2017). Moral disengagement strategies in sex offenders. Psychiatry, Psychology and Law, 24(3), 470-480. https://doi.org/10.1080/13218719.2016.1252291

Pelton, J., Gound, M., Forehand, R. \& Brody, G. (2004). The Moral Disengagement Scale: Extension with an American minority sample. Journal of Psychopathology and Behavioral Assessment, 26, 31-39. https://doi.org/10.1023/B:JOBA.0000007454.34707.a5 
Pozzoli, T., Gini, G. \& Vieno, A. (2012). Individual and Class Moral Disengagement in Bullying Among Elementary School Children. Aggressive Behaviour, 0, 1-11. https://doi.org/10.1002/ ab. 21442

Quinn, C. A. \& Bussey, K. (2015a). Moral disengagement, anticipated social outcomes and adolescents' alcohol use: Parallel latent growth curve analyses. Journal of Youth and Adolescence, 44, 1854-1870. https://doi.org/10.1007/s10964-015-0345-6

Quinn, C. A. \& Bussey, K. (2015b). The role of moral disengagement in underage drinking and heavy episodic drinking. Substance Use \& Misuse, 50, 1437-1448. https://doi.org/10.3109/10826084.2 015.1018541

Raskauskas, J. L., Gregory, J., Harvey, S. T., Rifshana, F. \& Evans, I. M. (2010). Bullying among primary school children in New Zealand: Relationships with prosocial behaviour and classroom climate. Educational Research, 52, 1-13. https://doi. org/10.1080/00131881003588097

Redondo, P, J., Rueda, R, S. \& Amado, V, C., (2013). Conducta prosocial: una alternativa a las conductas agresivas. Investigium ire, 4(1), 2, 234-247. Recuperado de http://investigiumire. iucesmag.edu.co/index.php/ire/article/viewFile/56/55

Redondo, J., Rangel, K. \& Luzardo, M. (2015). Diferencias en comportamientos prosociales entre adolescentes colombianos. Psicogente, 18(34), 311-319. https://doi.org/10.17081/psico.18.34.507

Retuerto, A. (2004). Diferencias en empatía en función de las variables género y edad. Apuntes de Psicología, 22(3), 323-339. Recuperado de http://www.apuntesdepsicologia.es/index.php/revista/ article/view/59/61

Richaud de Minzi, M. C. (2009). Influencia del modelado de los padres sobre el desarrollo del razonamiento prosocial en los/las niños/as. Interamerican Journal of Psychology, 43(1), 187-198. Recuperado de http://pepsic.bvsalud.org/scielo.php?script=sci_arttext\&pid= S0034-96902009000100021 
Richaud de Minzi, M.C. (2014). Algunos aportes sobre la importancia de la empatía y la prosocialidad en el desarrollo humano. Revista Mexicana de Investigación en Psicología, 6(2), 171-176. Recuperado de www.revistamexicanadeinvestigacionenpsicologia.com/ article/view/194/107

Richaud de Minzi, M.C., Lemos, V. \& Mesurado, B. (2011). Relaciones entre la percepción que tienen los niños de los estilos de relación y de la empatía de los padres y la conducta prosocial en la niñez media y tardía. Avances en Psicología Latinoamericana, 29(2), 330-343. Recuperado de http://www.scielo.org.co/scielo. php?script=sci_arttext\&pid=S1794-47242011000200012

Richaud de Minzi, M. C. \& Mesurado, B. (2016). Las emociones positivas y la empatía como promotores de las conductas prosociales e inhibidores de las conductas agresivas. Acción psicológica, 13(2), 31-42. https://doi.org/10.5944/ap.13.2.17808

Rodríguez, L. M., Mesurado, B., Ońate, M. E., Guerra, P. \& Menghi, M.S. (2017). Adaptación de la Escala de Prosocialidad de Caprara en adolescentes argentinos. Revista Evaluar, 17(2), 177-187. Recuperado de https://revistas.unc.edu.ar/index.php/ revaluar

Rubio-Garay, F., Amor, P. \& Carrasco, M. (2017). Dimensionality and psychometric properties of the Spanish version of the Mechanisms of Moral Disengagement Scale (MMDS-S). Revista de Psicopatología y Psicología Clinica, 22, 43-54. https://doi. org/10.5944/rppc.vol.22.num.1.2017.16014

Samper, P. (2014). Diferentes tendencias prosociales: el papel de las emociones. Revista Mexicana de Investigación Psicológica, 6(2), 177-185. Recuperado de https://core.ac.uk/download/pdf/ 71038302.pdf

Shulman, E. P., Cauffman, E., Piquero, A. R. \& Fagan, J. (2011). Moral disengagement among serious juvenile offenders: A longitudinal study of the relations between morally disengaged attitudes and offending. Developmental Psychology, 47(6), 1619-1632. http:// dx.doi.org/10.1037/a0025404 
Tur, P. A. (2003). Conducta Agresiva y prosocial en relación con temperamento y hábitos de crianza en niños y adolescentes. Valencia: Proquest.

Villegas de Posada, C., Flórez, J. \& Espinel, N. (2018). Moral disengagement mechanisms and armed violence. A comparative study of paramilitaries and guerrillas in Colombia. Revista Colombiana de Psicología, 27, 55-69. https://doi.org/10.15446/ rcp.v27n1.6219

Williford, A., Boulton, A.J., Forrest-Bank, S.S., Bender, K.A., Dieterich, W.A. \& Jenson, J.M. (2015). The effect of bullying and victimization on cognitive empathy development during the transition to Middle School. Child \& Youth Care Forum, 45, 525-541. https://doi.org/10.1007/s10566-015-9343-9

Van Noorden, T.H., Haselager, G. J., Cillessen, A.H. \& Bukowski, W.M. (2014). Dehumanization in children: The link with moral disengagement in bullying and victimization. Aggressive Behavior, 40, 320-328. https://doi.org/10.1002/ab.21522

Recibido: 27 de agosto, 2018

Revisado: 14 de febrero, 2019

Aceptado: 1 de abril, 2019 